\title{
Modeling of distortions induced by the nitriding process
}

\author{
P. Depouhon ${ }^{1,2, a}$, J.-M. Sprauel ${ }^{1}$, M. Mailhé ${ }^{2}$ and E. Mermoz ${ }^{2}$ \\ 1 Aix-Marseille Université, CNRS, ISM UMR 7287, 13288 Marseille Cedex 09, France \\ 2 Airbus Helicopters, Aéroport international Marseille Provence, 13725 Marignane, France
}

Received 1 July 2014, Accepted 16 July 2014

\begin{abstract}
Nitriding is a thermochemical process that is used to improve the fatigue life of structural parts. It also involves residual stresses and non-negligible distortions of treated parts. This work deals with a pre-dimensioning tool able to simulate distortions of complex parts, generated during treatment. The presented model uses the analogy between diffusion of species and heat conduction to describe development of apparent volumic eigenstrains of the treated material, which is the source of global part distortions. The first step of the model consists in identifying these apparent volumic eigenstrains using experimental distortion measurements of a nitrided sample. Then, the unstationnary equivalent boundary condition of an equivalent diffusion problem is developed in order to numerically generate the volumic strains previously identified. Finally, this equivalent boundary condition is integrated in a heat conduction finite element code to simulate distortions of parts with complex geometries. In case of an industrial sample, the predicted distortions are compared to experimental measurements performed on a 3D coordinate measuring machine. The presented tool allows evaluating the consequences of a design choice on the final part distortions induced by the treatment.
\end{abstract}

Key words: Nitriding / modeling / distortions / finite element method

\section{Introduction}

Thermochemical treatments such as nitriding are usually used to improve the fatigue life of structural parts. Benefits of residual stresses generated by this kind of treatment is well-known [1-3]. Many studies have been performed to describe the microstructure of nitrided layers [4-6]. Several are available to model residual stress development $[7,8]$, but few deal with the distortion of mechanical parts. However, in the aeronautic industry, the mass of structural parts tends to be constantly reduced. This specificity implies the design of thinner parts, which are more favorable to distortions. These distortions, usually neglected, have to be mastered in order to ensure the compliance of treated parts.

The nitriding process involves complex multi-physics phenomena at the source of distortions. In this work, the case study is the gas nitriding of $32 \mathrm{CrMoV} 13$ low alloy steel treated during $120 \mathrm{~h}$. In this kind of steel, nitrogen atoms diffuse from the surface and interact with iron and alloying elements [9-14]. Nitrides are formed from initial carbides or alloying elements in iron solid solution. Carbon atoms released during the dissolution of

\footnotetext{
a Corresponding author:

pierre.depouhon@airbus.com
}

initial carbides can diffuse in the depth and form new carbides such as cementite or be evacuated from the steel, which involves decarburizing on the surface [15]. This microstructure evolution involves volume changes of nitrided layers, resulting in macroscopic residual stresses and distortions [7].

This work focuses on the mechanical aspect of the treatment. It allows providing a pre-dimensioning tool, whose computational time is reduced while simulating complex geometrical parts. Volumic eigenstrains (accompanying precipitations) are considered as the source of residual stresses and distortions. In the model, the volumic strains are identified from the curvature of a thin plate nitrided on one face [16] using the radius curvature method.

Once the volumic strains are identified, they are imposed numerically to the studied structure thanks to an equivalent diffusion problem. Indeed, a volumic strain is considered as a dilatation accompanying the diffusion of an equivalent quantity. The main assumption consists in considering that volumic strains generated by nitrogen and carbon diffusion coupled with precipitation could be obtained by the diffusion of a unique equivalent quantity generating volume changes calculated as equivalent thermal expansion. The benefit of this approach lies in its simplicity, its reduced computational time and its instant 


\section{Nomenclature}

\begin{tabular}{|c|c|}
\hline Symbol & Definition \\
\hline$a, b, . ., g$ & Polynomial constant coefficients \\
\hline$a_{d}$ & Equivalent numerical diffusivity coefficient \\
\hline$C$ & $\begin{array}{l}\text { Concentration of the diffusing equivalent } \\
\text { quantity }\end{array}$ \\
\hline$C_{\text {ext }}$ & External equivalent quantity \\
\hline$e$ & Thickness of the removed layer (mm) \\
\hline$H$ & Thickness of the plate sample (mm) \\
\hline$h$ & Equivalent numerical transfert coefficient \\
\hline$I$ & Identity tensor \\
\hline$I$ & Cost function (curvature identification) \\
\hline$J$ & Cost function (volumic strain identification) \\
\hline$K$ & Hooke's tensor \\
\hline$q$ & Flux of the equivalent quantity \\
\hline$R$ & Curvature radius $(\mathrm{m})$ \\
\hline$T$ & Pseudo-period of the function $C_{e x t}(t)$ \\
\hline$t$ & Time (s) \\
\hline$t_{1}$ & Final time of the equivalent diffusion (s) \\
\hline$u$ & Displacement vector $(\mu \mathrm{m})$ \\
\hline$\nabla u$ & Displacement gradient tensor \\
\hline$x$ & 3D Coordinates of a matter point $(\mathrm{mm})$ \\
\hline$z$ & In-depth abscissa $(\mathrm{mm})$ \\
\hline$z_{F}$ & Abscissa of the end of the nitrided layer \\
\hline$z_{M}$ & Abscissa of $\varepsilon_{M}$ \\
\hline$\alpha$ & Equivalent numerical dilatation coefficient \\
\hline$\varepsilon$ & Total strain tensor \\
\hline$\varepsilon_{e}$ & Elastic strain tensor \\
\hline$\varepsilon_{f}$ & Apparent volumic strain tensor \\
\hline$\varepsilon_{f}$ & Apparent volumic strain such as $\varepsilon_{f}=\varepsilon_{f} \boldsymbol{I}$ \\
\hline$\varepsilon_{f}^{\text {diff }}$ & $\begin{array}{l}\text { Apparent volumic strain after numerical } \\
\text { diffusion }\end{array}$ \\
\hline$\varepsilon_{M}$ & Maximum apparent volumic strain \\
\hline$\varepsilon_{0}$ & Apparent volumic strain in surface $(z=0)$ \\
\hline$\lambda$ & Equivalent numerical conductivity \\
\hline$\sigma$ & Residual stress (Cauchy stress tensor) (MPa) \\
\hline$\chi$ & Theoretical curvature $\left(\mathrm{m}^{-1}\right)$ \\
\hline$\chi^{\exp }$ & Experimental curvature $\left(\mathrm{m}^{-1}\right)$ \\
\hline$\chi_{a d}^{\exp }$ & Adjusted experimental curvature $\left(\mathrm{m}^{-1}\right)$ \\
\hline$\chi_{0}$ & Initial experimental curvature $\left(\mathrm{m}^{-1}\right)$ \\
\hline$\chi_{p}$ & Residual experimental curvature $\left(\mathrm{m}^{-1}\right)$ \\
\hline$\Omega$ & Domain occupied by the studied solid \\
\hline$\partial \Omega$ & Boundary of the domain $\Omega$ \\
\hline
\end{tabular}

integration in usual finite element methods where heat conduction equations are implemented. It allows understanding the link between the shape of parts and their distortions and enables to compute an anticipated geometry which improves the compliance of treated parts.

\section{Mechanical approach of the treatment}

The aim of this study being the prediction of distortions, the constitutive law is supposed to be linear elastic. Therefore, visco-plastic phenomena are neglected.

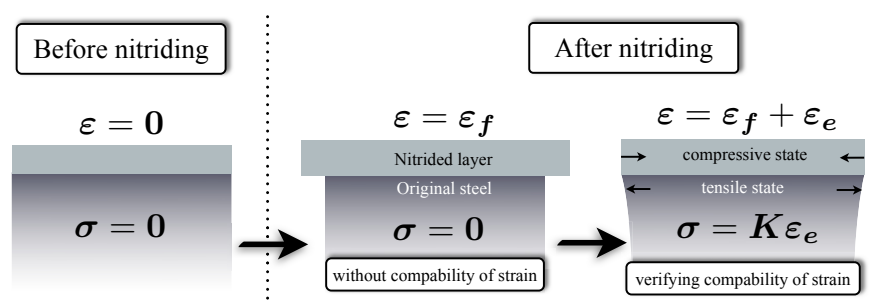

Fig. 1. Schematic approach of the generation of residual stresses and distortions.

The mechanical problem is to find $(\boldsymbol{u}, \boldsymbol{\sigma})(\boldsymbol{x})$ sufficiently regular at every point $\boldsymbol{x}$ of the domain $\Omega$ occupied by the studied solid ( $\partial \Omega$ is its boundary) [17] such as:

$$
\begin{cases}\text { Kinematic compatibility: } & \text { Equation of equilibrium: } \\ \boldsymbol{\varepsilon}=\frac{1}{2}\left(\boldsymbol{\nabla} \boldsymbol{u}^{T}+\boldsymbol{\nabla} \boldsymbol{u}\right) & \operatorname{div}(\boldsymbol{\sigma})=\mathbf{0} \\ \varepsilon=\varepsilon_{\boldsymbol{e}}+\varepsilon_{\boldsymbol{f}} & \text { Constitutive law: } \\ \varepsilon_{\boldsymbol{f}}=\varepsilon_{f} \boldsymbol{I} & \boldsymbol{\sigma}=\boldsymbol{K} \varepsilon_{\boldsymbol{e}}\end{cases}
$$

with $\boldsymbol{u}$ the displacement field, $\boldsymbol{\sigma}$ the Cauchy stress tensor and $\boldsymbol{K}$ Hooke's tensor. $\boldsymbol{\varepsilon}$ is the total strain tensor, $\varepsilon_{e}$ the elastic strain, and $\varepsilon_{f}$ apparent volumic eingenstrain generated by nitriding. It is considered purely hydrostatic due to quasi-random distribution of nitrides in martensite. Assuming that the infinitesimal strain theory is verified, the total strain can be separated in summation of an elastic and an apparent volumic strain [17]. Figure 1 schematically shows the generation of residual stresses and distortions, caused by a volumic strain mismatch.

The presented approach considers that the only consequence of the nitriding is the imposed apparent volumic eigenstrains $\varepsilon_{f}$. The elastic strain $\varepsilon_{e}$ is then an accommodation generated to guarantee the compatibility of the total strain $\varepsilon$, which is defined as the symmetrical gradient of displacements (Eq. (1)).

\section{Apparent volumic strain identification}

Identification of the apparent volumic strain is performed using the experimental results provided by Goret et al. [16].

They used the curvature radius method to evaluate the in-depth residual stress profile. The experiment consists in nitriding a thin plate only on one face in order to generate a bending moment which curves it (Fig. 2). Successive material removals are then performed using chemical etching and the evolution of the curvature radius $R$ (assumed to be constant at any point of the plate) is measured using a $3 \mathrm{D}$ coordinate measuring machine.

Figure 3 shows the experimental curvature $\chi^{\exp }=$ $\frac{1}{R^{\exp }}$ of the plate in respect of the removed material thickness. 


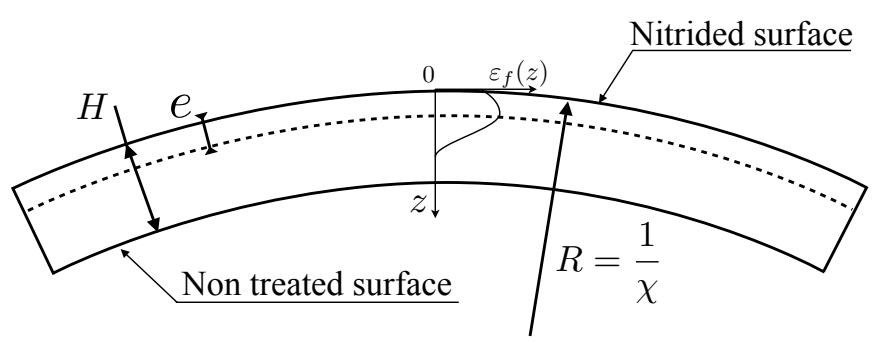

Fig. 2. Drawing of a thin plate once nitrided on one face.

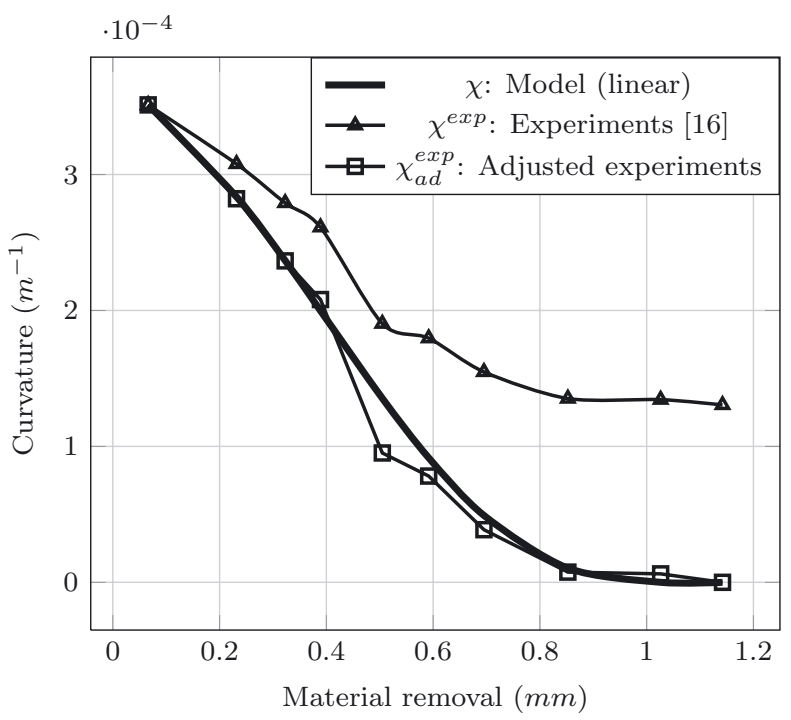

Fig. 3. Curvature of a thin plate nitrided on one face in respect to removed thickness [16].

The theoretical curvature $\chi=\frac{1}{R}$ in respect to the removed material thickness $e$ and the apparent volumic strain $\varepsilon_{f}$ is also plotted. In case of thin plate geometry ( $H$ its thickness, $z$ the depth in the nitrided layer), it can be expressed:

$$
\begin{aligned}
\chi\left(e, \varepsilon_{f}\right) & =\frac{1}{R\left(e, \varepsilon_{f}\right)} \\
& =\frac{6(H+e) \int_{e}^{H} \varepsilon_{f}(z) \mathrm{d} z+2 \int_{e}^{H} z \varepsilon_{f}(z) \mathrm{d} z}{(H-e)^{3}}
\end{aligned}
$$

The experimental results (Fig. 3) show a remaining residual curvature even after the total removal of the nitrided layer (depth over $1.1 \mathrm{~mm}$ ). It means that plastic and visco-plastic effects appear in the non-treated material during the treatment. The considered model does not account for these phenomena, and expects a zero curvature once the nitrided layer is removed. This explains the significant discrepancy between the experimental results and the model predictions.

To account for this impediment, an adjusted experimental curvature is defined such as:

$$
\chi_{a d}^{\exp }=\chi_{0} \frac{\chi^{\exp }-\chi_{p}}{\chi_{0}-\chi_{p}}
$$

where $\chi_{a d}^{\exp }$ is the adjusted experimental curvature, $\chi_{0}$ the initial curvature before etching and $\chi_{p}$ the residual curvature after removal of the nitrided layer. In this expression, $\chi_{p}$ is linked to visco-plastic strains induced by the bending moment appearing to satisfy the global equilibrium of the plate. This residual curvature is subtracted to the experimental curve and redistributed in order to obtain a null curvature once the nitrided layer is totally removed and to fit the real initial curvature before etching $\left(\chi_{0}\right)$. This permits defining $\varepsilon_{f}(z)$ as an apparent volumic strain which is not only related to microstructure volume changes, but also accounts (in a global manner) for plastic and visco-plastic effects.

The identification problem consists thus in finding the apparent volumic strain which provides the best fitting between the theoretical and the adjusted experimental curvatures $\chi$ and $\chi_{a d}^{\exp }$. In order to reduce the solution search space, the in-depth apparent volumic strain profile is assumed to be piecewise polynomial (Fig. 4) such as:

$$
\begin{aligned}
\varepsilon_{f}(z) & =\varepsilon_{f}\left(z, \varepsilon_{0}, \varepsilon_{M}, z_{M}, z_{F}\right) \\
& = \begin{cases}a z^{2}+b z+c & \forall z \in\left[0 ; z_{M}\right] \\
d z^{3}+e z^{2}+f z+g & \forall z \in\left[z_{M} ; z_{F}\right]\end{cases}
\end{aligned}
$$

where $(a, b, c, d, e, f, g)$ are constants which depend on $\left(\varepsilon_{0}, \varepsilon_{M}, z_{M}, z_{F}\right)$. The function $\varepsilon_{f}(z)$ and its derivative are built to be continuous. The selected profile (Fig. 4) is based on phenomenological considerations. Indeed, decarburizing involves a lower volumic strain at surface than in the depth, thus $\varepsilon_{0} \leq \varepsilon_{M}$ is imposed [8]. Maximum point $\left(z_{M}, \varepsilon_{M}\right)$ is directly linked to the maximum compression stress level observed around $300 \mu \mathrm{m}$ in the depth [16]. At last, considering an analogy with a real diffusion profile, the curve has a tangent to zero in $z_{F}$, corresponding to the end of the diffusion front. The polynomial degree of the apparent volumic strain profile (Eq. (4)) is selected in order to correspond to the shape of the residual stress profile (Fig. 4).

Thus, the identification of apparent volumic strain is to find the parameters $\left\{\varepsilon_{0}, \varepsilon_{M}, z_{M}, z_{F}\right\}$ minimizing the cost function $I$ defined in the least squares sense by:

$$
I=\sum_{k=1}^{N}\left[\chi_{a d}^{\exp }\left(e_{k}\right)-\chi\left(e_{k}, \varepsilon_{0}, \varepsilon_{M}, z_{M}, z_{F}\right)\right]^{2}
$$

with $\chi_{a d}^{\exp }\left(e_{k}\right)$ the adjusted experimental curvature at $e_{k}$ depth of $k^{\text {st }}$ material removal and $N$ the number of measurement points taken into account in the minimization problem $(N=10)$. The simplex optimization algorithm is used to determine the four optimal parameters [18].

\section{Equivalent diffusion problem}

Once the apparent volumic strain is calculated, it is considered as the solution of a one dimensional equivalent diffusion problem. A convection boundary condition 


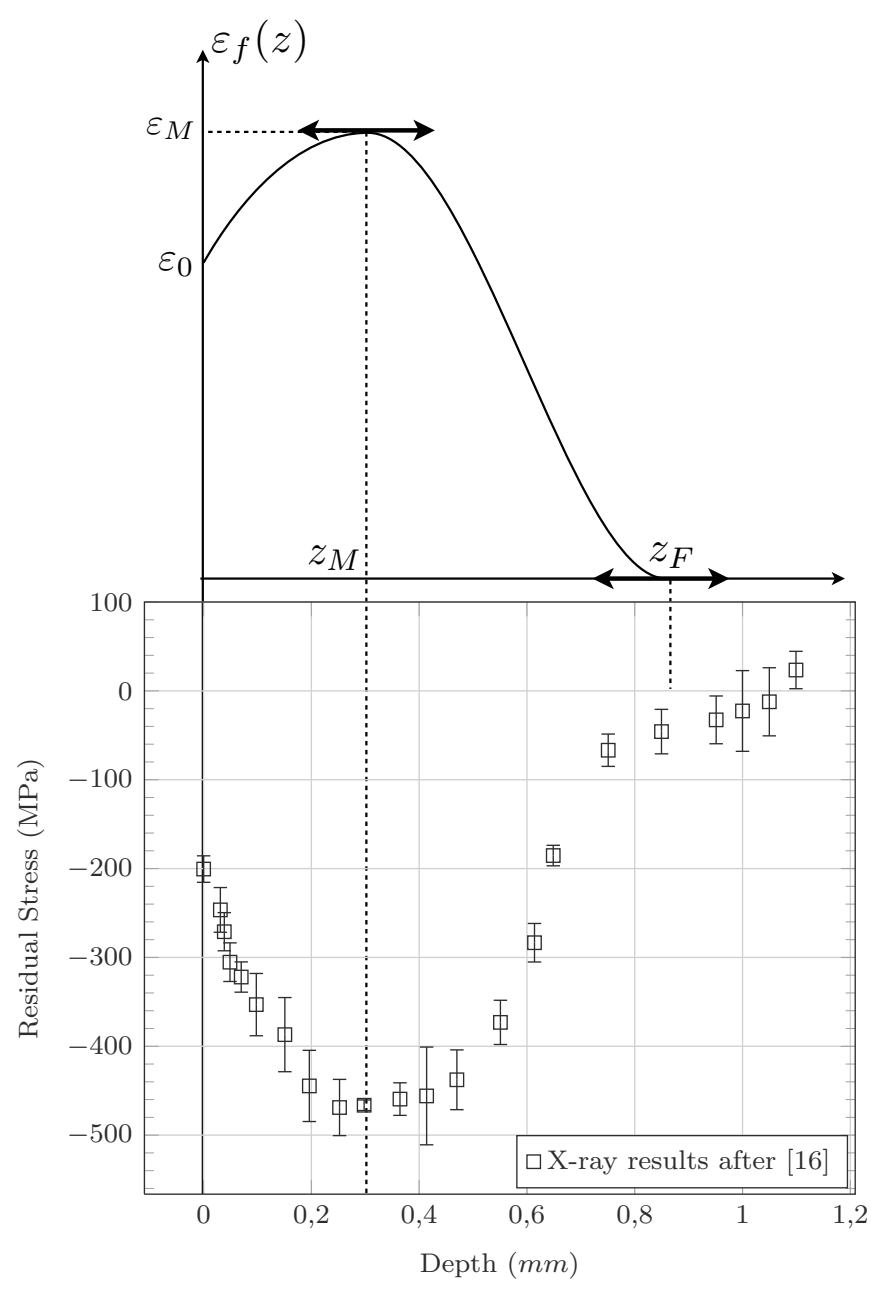

Fig. 4. Theoretical in-depth apparent volumic strain profile, assumed to be piecewise polynomial, built in respect to the in-depth residual stress profile.

is chosen. The diffusion problem is to find $(\mathrm{C}, q)$ sufficiently regular in $\Omega=[0 ; H]$ such as:

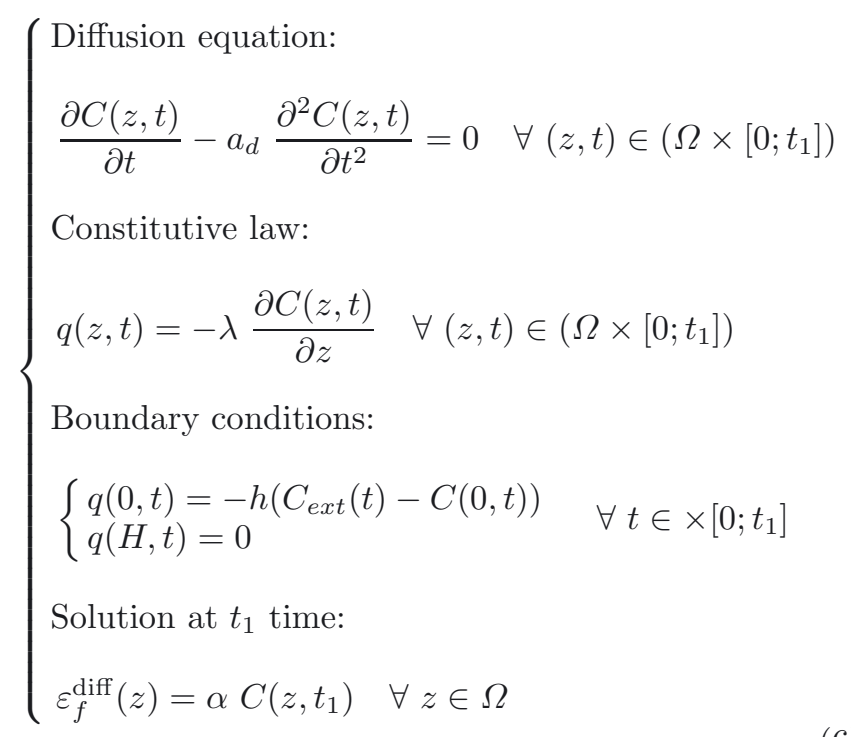

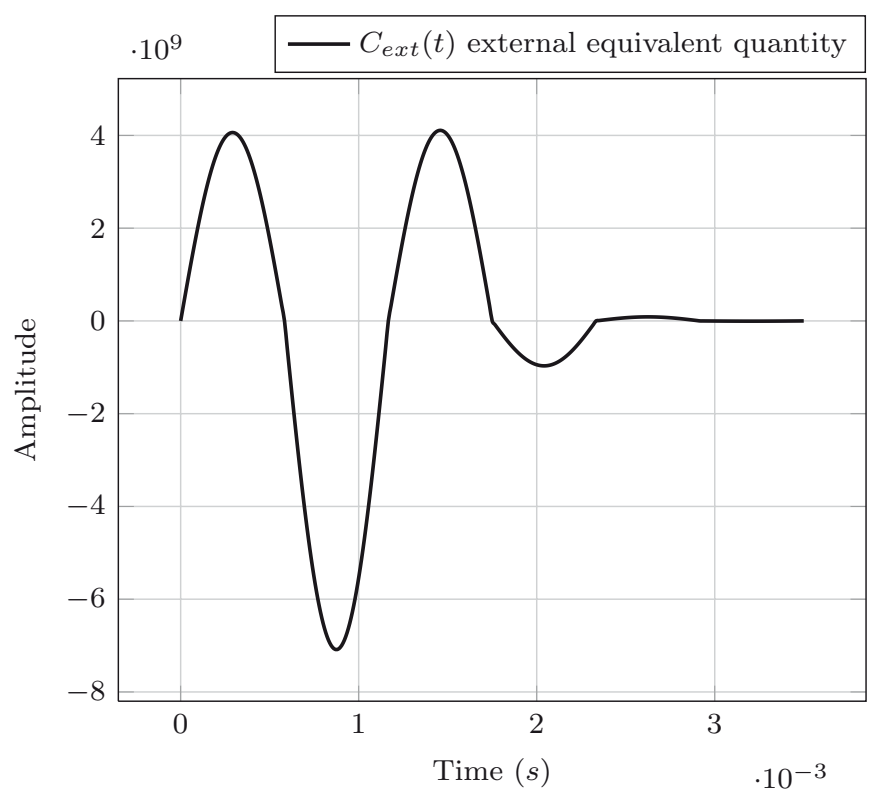

Fig. 5. External equivalent quantity which is the condition numerically applied to the surface layer of the treated material.

where $C(z, t)$ is the concentration of a diffusing equivalent quantity and $q$ its flux. $a_{d}, \lambda, h, \alpha$ are constants and $t_{1}$ is a given time corresponding to the end of the simulation. The direct problem (one dimensional diffusion) is solved using Crank-Nicolson numerical scheme [19].

\section{Identification of an external equivalent quantity}

The inverse problem consists in finding $C_{\text {ext }}(t)$ (an unstationary external equivalent quantity which gives $\left.\varepsilon_{f}^{\text {diff }}(z)=\alpha C\left(z, t_{1}\right)\right)$ such as $\varepsilon_{f}^{\text {diff }}(z)$ is as close as possible to $\varepsilon_{f}(z)$, previously identified.

In order to reduce the solution search space, $C_{\text {ext }}(t)$ belongs to the family of piecewise T-periodic sinusoidal functions such as $T=\frac{N_{s}}{2 t_{1}} . N_{s}$ is the number of sinusoidal segments on the time interval $\left[0 ; t_{1}\right]$. For each segment, the only unknown is the sinus amplitude $C_{i}^{\max }$ where $i \in$ $\llbracket 1 ; N_{s} \rrbracket$. Finally, the optimization problem is to find the parameters $\left(t_{1}, N_{s},\left(C_{i}^{\max }\right)_{1 \leq i \leq N_{s}}\right)$ which minimize the cost function:

$$
J=\int_{0}^{H}\left[\varepsilon_{f}(z)-\varepsilon_{f}^{\operatorname{diff}}(z)\right]^{2} \mathrm{~d} z
$$

Simplex algorithm is used in the same manner as for the identification of apparent volumic strain.

Figure 5 shows an optimal external unstationnary equivalent quantity $C_{\text {ext }}^{\text {opti }}(t)$ minimizing (locally) the cost function $J$. At time $t_{1}$, the apparent volumic strain generated by the numerical diffusion with the surface condition $C_{\text {ext }}^{\text {opti }}(t)$ is shown and compared to $\varepsilon_{f}(z)$ in Figure 6 . The shape of the experimentally identified apparent volumic strain profile $\varepsilon_{f}(z)$ is well fitted by the optimal $\varepsilon_{f}^{\text {diff }}(z)$. 


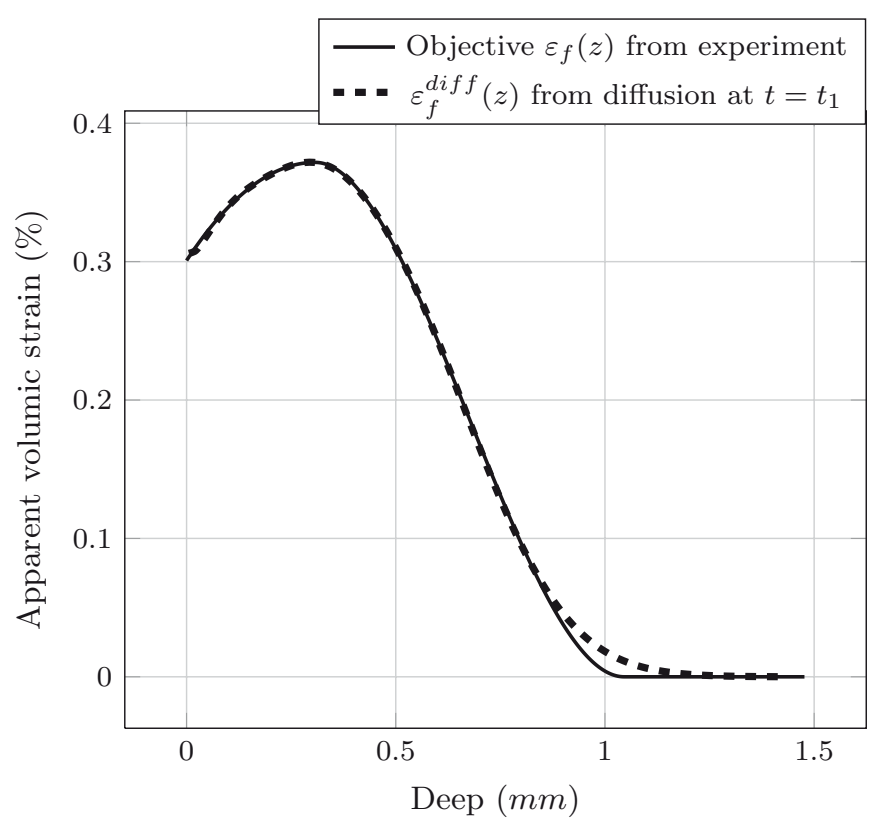

Fig. 6. Comparison of in-depth apparent volumic strain resulting from numerical diffusion at $t=t_{1}$ and from experimental identification.

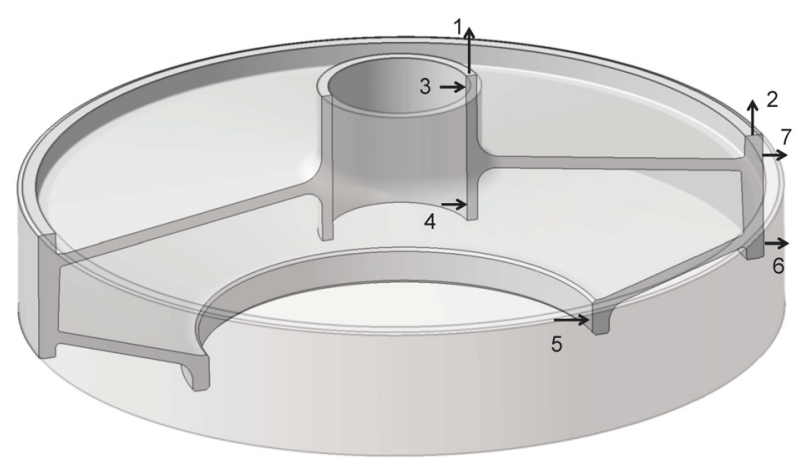

Fig. 7. Industrial case for validation. All surfaces are nitrided. Landmarks 1 to 7 correspond to the measurement results shown in Figure 9 (Design details are suppressed).

\section{Results}

Once the external equivalent quantity $C_{\mathrm{ext}}^{\mathrm{opti}}(t)$ identified, it is numerically applied on each "nitrided" surface of a finite element mesh. In order to validate the model, an industrial complex geometry case is simulated using an industrial finite element code. The predicted distortions are then compared to experimental results obtained by a $3 \mathrm{D}$ coordinate measuring machine. Figure 7 shows the geometry of the case study. It is a simplified large gear that presents a complex shape section. Its geometry allows to performed axisymmetric computation. Figure 8 presents the simulated distortions. Naturally, it shows that distortions are largely influenced by the initial part geometry. Massive zones are less distorted than the thin ones. In our case, axial displacements are twice the radial ones. Figure 9 compared the predicted axial and radial distortions to the values measured at seven points. These

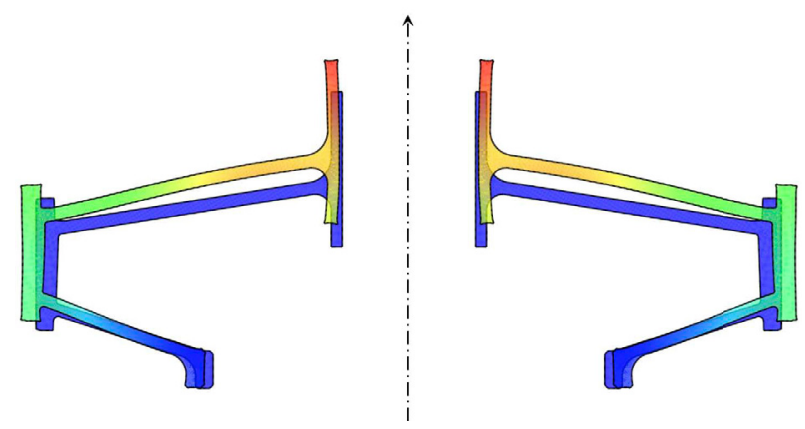

Fig. 8. Theoretical distortions. Blue section is the undeformed geometry before treatment; in color, the geometry after treatment $(\times 100)$. Axial and radial displacements are shown in Figure 9 .

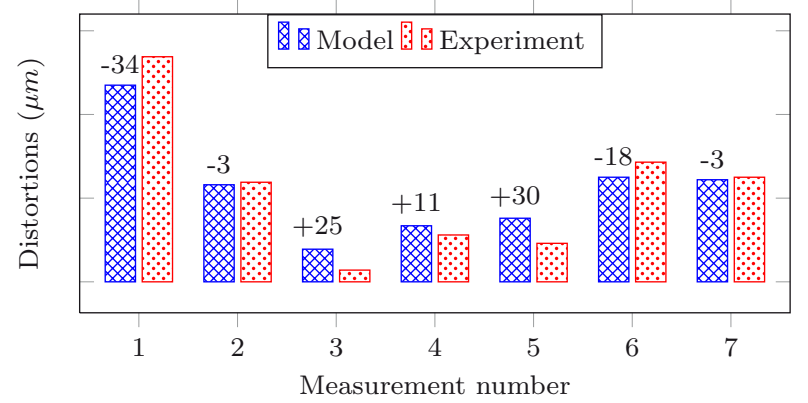

Fig. 9. Comparison of model predictions and experimental distortions measured at 7 points. The numbers above model bars are the absolute discrepancy between experiments and model $(\mu \mathrm{m})$.

measurements (part geometry before and after nitriding) have been carried out in the zones defined in Figure 7. Measures 1 and 2 correspond to axial displacements. The maximum absolute discrepancy is about $34 \mu \mathrm{m}$, and the relative one about $13 \%$. Measures 3 to 7 correspond to radial displacements, with a maximum absolute discrepancy about $32 \mu \mathrm{m}$. The relative discrepancy becomes non relevant because of the low level of displacements compared to the measurement uncertainty.

Considering the large size of the studied sample (several decimeters), the predicted distortions are in very good agreement with the experimental data.

\section{Conclusions}

This work presented a simplified model for the prediction of structural part distortions generated by nitriding. Our results showed that these deformations could be accurately related to apparent volumic eigenstrains. These eigenstrains, generated during nitriding, were assumed to be the only source of distortions. They could be identified from a nitrided plate and then simulated thanks to an equivalent diffusion problem. Furthermore, the numerical external equivalent condition identified in the latter diffusion problem was supposed to characterize the distortions generated by a given set of nitriding parameters 
applied to a fixed material. Thus, only one nitrided plate was needed to identify the numerical external equivalent condition to be imposed to future simulations. It allows predicting distortions for any complex geometry for the same fixed material and nitriding parameters. The results of such simulations were compared to experimental measurements and gave a good estimation of the real distortions. Thus, our simulation method proved to be a good pre dimensioning tool to anticipate distortions. Moreover, the presented approach is not limited to nitriding (because there is no description of nitriding specific micro structural transformation) but could enable to model distortions of any phenomena generating volume changes such that the macroscopic free strain is hydrostatic.

\section{References}

[1] H.C.F. Rozendaal, E.J. Mittemeijer, P.F. Colijn, P.J. Van Der Schaaf, The development of nitrogen concentration profiles on nitriding iron, Metall. Mater. Trans. A 14 (1983) 395-399

[2] C.X. Li, Y. Sun, T. Bell, Consideration of fretting fatigue properties of plasma nitrided steel: second prize, Surface Engineering, Maney Publishing, 1999, pp. 149-153

[3] S. Aissat, A. Iost, G. Guillemot, Y. Benarioua, M. Mechmeche, Caractérisation des propriétés mécaniques par nanoindentation d'un traitement de diffusion et d'un revêtement pour l'amélioration de la résistance à l'usure des aciers à bas carbone, Mécanique \& Industries, Cambridge Univ. Press, 2011, Vol. 12, pp. 379-387

[4] R.E. Schacherl, P.C.J. Graat, E.J. Mittemeijer, The nitriding kinetics of iron-chromium alloys, the role of excess nitrogen: Experiments and modelling, Metall. Mater. Trans. A 35 (2004) 3387-3398

[5] S.S. Hosmani, R.E. Schacherl, E.J. Mittemeijer, Nitrogen uptake by an Fe-V alloy: Quantitative analysis of excess nitrogen, Acta Mater. 54 (2006) 2783-2792

[6] H.P. Van Landeghem, M. Gouné, A. Redjaïmia, Nitride precipitation in compositionally heterogeneous alloys: Nucleation, growth and coarsening during nitriding, J. Cryst. Growth 341 (2012) 53-60
[7] M.A.J. Somers, E.J. Mittemeijer, Development and relaxation of stress in surface layers; Composition and residual stress profiles in $\gamma^{\prime}-\mathrm{Fe}_{4} \mathrm{~N}_{1-x}$ layers on $\alpha$-Fe substrates, Metall. Mater. Trans. A 21 (1990) 189-204

[8] S. Jegou, L. Barrallier, R. Kubler, Phase transformations and induced volume changes in a nitrided ternary Fe-3\% Cr-0.345\% C alloy, Acta Mater. 58 (2010) 2666-2676

[9] Y. Sun, T. Bell, A numerical model of plasma nitriding of low alloy steels, Mater. Sci. 224 (1997) 33-47

[10] E.J. Mittemeijer, J.T. Slycke, Chemical potentials and activities of nitrogen and carbon imposed by gaseous nitriding and carburising atmospheres, Surf. Eng. 12 (1996) $152-162$

[11] T. Belmonte, M. Gouné, H. Michel, Numerical modeling of interstitial diffusion in binary systems, Application to iron nitriding, Mater. Sci. Eng. A 302 (2001) 246-257

[12] M. Keddam, M.E. Djeghlal, L. Barrallier, A diffusion model for simulation of bilayer growth $(\varepsilon / \gamma)$ of nitrided pure iron, Mater. Sci. Eng. A 378 (2004) 475-478

[13] R. Kouba, M. Keddam, M.E. Djeghlal, A Diffusionprecipitation Model for gaseous nitriding of Fe-2 Wt.\% V alloy, J. Alloys Compd. 536 (2012) 124-131

[14] P. Cavaliere, G. Zavarise, M. Perillo, Modeling of the carburizing and nitriding processes, Comput. Mater. Sci. 46 (2009) 26-35

[15] S. Jegou, Influence des éléments d'alliage sur la genèse des contraintes résiduelles d'aciers nitrurés, Thèse, Arts et Métiers ParisTech, 2009

[16] L. Barrallier, V. Goret, P. Vardon, D. Deloison, Residual stresses and distortion simulation of nitrided disc, JCPDS-International Centre for Diffraction Data, 2009

[17] J. Lemaitre, J.L. Chaboche, A. Benallal, R. Desmorat, Mécanique des matériaux solides, 3ème édition, Dunod, 2009

[18] G.B. Dantzig, A. Orden, P. Wolfe, The generalized simplex method for minimizing a linear form under linear inequality restraints, Pacific J. Math. 5 (1955) 183-195

[19] J. Crank, The Mathematics of Diffusion, Clarendon Press, Oxford, 1956 